\title{
Is irrational use of intralipid emulsion justified in amlodipine toxicity?
}

\author{
Bhavna Gupta and Sukhyanti Kerai \\ Department of Anesthesia and Critical Care, Maulana Azad Medical College and Lok Nayak Hospital, New Delhi, \\ India
}

In reference to the letter to the editor by $\mathrm{Ok}$ and Sohn [1] highlighting the importance of intra-lipid emulsion (ILE) in amlodipine toxicity, suggested for the management of a case of amlodipine toxicity with concurrent medications, published in the Korean Journal of Anesthesiology [2], we would like to highlight a few points related to the effectiveness of ILE in amlodipine toxicity. Intra-lipid $20 \%$ is an intravenous emulsion of fat, administered to patients requiring parenteral nutrition as a source of calories and essential fatty acids. Its role in management of life-threatening cardiac arrest because of local anesthetic (especially bupivacaine) toxicity is well-documented; however, its role in the management of toxicity due to other lipophilic drugs, such as non-local anesthetic drugs, amitriptyline, calcium channel blockers, and baclofen, is not well-established, and most of the literature is based on published case reports [3].

Amlodipine toxicity is associated with significant morbidity and mortality, and the recommended first line therapy for symptomatic patients with calcium channel blocker (CCB) toxicity includes intravenous calcium, high dose of insulin in case of myocardial dysfunction, nor-epinephrine or epinephrine for vasodilatory shock, dobutamine for cardiogenic shock, and atropine in case of symptomatic bradycardia or conduction disturbances. Intravenous calcium is the recommended first line

Corresponding author: Bhavna Gupta, MBBS., DA, DNB.

Department of Anesthesia and Critical Care, Maulana Azad Medical College and Lok Nayak Hospital, Bahadur Shah Zafar Marg, Balmiki Basti, New Delhi 110002, India

Tel: 91-8527686660, Fax: 91-1126853108

Email: bhavna.kakkar@gmail.com

ORCID: https://orcid.org/0000-0002-3108-0408

Received: June 4, 2018.

Accepted: June 24, 2018.

Korean J Anesthesiol 2019 April 72(2): 191-193

https://doi.org/10.4097/kja.d.18.00152 treatment as it improves contractility and blood pressure, carries little risk, and is a readily available drug. High dose of insulin is also known to improve contractility, increase blood pressure, and improve survival, according to observational studies, case series, and animal studies [4]. Recommendation to use ILE is neutral in cases of cardiac arrest due to CCB toxicity (diltiazem, verapamil, and dihydropyridines); ILE should not be used as the first line therapy in life-threatening or non-life-threatening toxicity due to CCBs (level of recommendation: 2D) [3]. The rationale of not using ILE as first line in CCB toxicity is because of inconsistent reported outcomes, including sudden deaths as immediate response, observed in human case reports and animal experiments; therefore, no clear recommendation of its use exists [3]. There are frequent known adverse effects of ILE which occur as a result of contamination of intravenous lines and vein irritation, both of which are not life-threatening. However, less frequent immediate reactions have been reported, including dyspnea; cyanosis; allergic reactions; headache; increase in temperature; chest-pain; flushing; dizziness; and delayed reactions such as jaundice, thrombocytopenia, focal seizure, splenomegaly, and shock [3]. Its administration is contraindicated in patients with disturbance of normal fat metabolism, such as hyperlipidemia and lipoid nephrosis [4]. Moreover, it has been postulated that ILE can enhance intestinal absorption of other toxins, thereby enhancing their effects, as suggested by oral drug poisoning models [4]. Concern has also been raised that concurrent administration of ILE with extracorporeal assist devices interferes with resuscitative medications. A study has also demonstrated worse outcome with verapamil when ILE was administered [5]. Although ILE is not recommended as the first line therapy, it is reserved as rescue therapy in refractory cases which cannot be managed with supportive measures. Step-wise management in case of CCB toxicity is summarized in Fig. 1, as adapted from "Experts consensus recommendations for management of calcium channel blocker poisoning in adults" [4]. Administration

(c) This is an open-access article distributed under the terms of the Creative Commons Attribution Non-Commercial License (http://creativecommons.org/ licenses/by-nc/4.0/), which permits unrestricted non-commercial use, distribution, and reproduction in any medium, provided the original work is properly cited. 


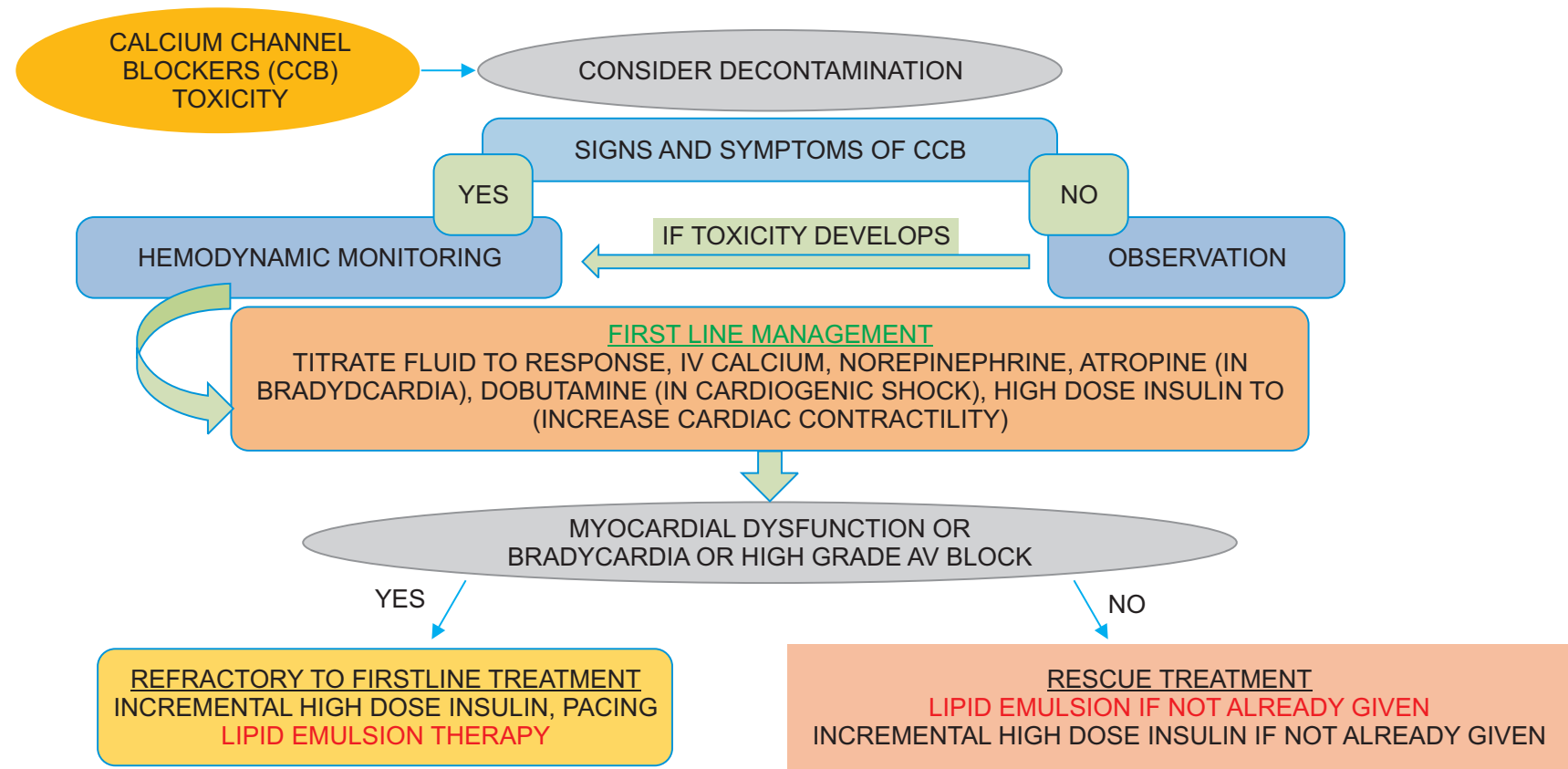

Fig. 1. Step-wise management in case of calcium channel blocker toxicity (adapted from Experts consensus recommendations for management of calcium channel blocker poisoning in adults).

of ILE is reserved only for refractory and rescue cases of CCB toxicity, and never used as the first modality of management. Therefore, unless organ perfusion is compromised, ILE may be reserved as a last resort treatment, although the question still prevails whether it should be a part of treatment modalities after standard therapy prevails. Certain signs and symptoms of CCB toxicity, such as bradycardia, hypotension, and ileus, may not respond to various therapies, but may rather be resolved with time as the toxicant is metabolized; hence, waiting and then proceeding step-by-step should be a norm in treating patients with suspected drug toxicity [4].

Although ILE has provided an exciting avenue for managing severe drug toxicity, with survival benefits, most of the evidence is from case reports, subject to positive reporting bias and confounding variables. Irrational use of ILE as a first line treatment is, however, not advocated in CCB toxicity. It may be considered as a last resort treatment when appropriately high supportive management is provided and the patient's condition continues to be unstable or there are no further conventional therapy options, as a rescue modality only.

\section{Conflicts of Interest}

No potential conflict of interest relevant to this article was reported.

\section{Author Contributions}

Bhavna Gupta (Conceptualization; Data curation; Investigation; Methodology)

Sukhyanti Kerai (Conceptualization)

\section{ORCID}

Bhavna Gupta, https://orcid.org/0000-0002-3108-0408

Sukhyanti Kerai, https://orcid.org/0000-0002-7771-0786

\section{References}

1. Ok SH, Sohn JT. Amlodipine toxicity and lipid emulsion. Korean J Anesthesiol 2018; 71: 491-2.

2. Gupta B, Kerai S. Amlodipine toxicity complicated by concurrent medications. Korean J Anesthesiol 2018; 71: 489-90.

3. Gosselin S, Hoegberg LC, Hoffman RS, Graudins A, Stork CM, Thomas SH, et al. Evidence-based recommendations on the use of intravenous lipid emulsion therapy in poisoning. Clin Toxicol (Phila) 2016; 54: 899-923.

4. St-Onge M, Anseeuw K, Cantrell FL, Gilchrist IC, Hantson P, Bailey B, et al. Experts consensus recommendations for the management of 
calcium channel blocker poisoning in adults. Crit Care Med 2017; 45: e306-15.

5. Perichon D, Turfus S, Graudins A. Intravenous lipid emulsion does not improve hemodynamics or survival in a rodent model of oral verapamil poisoning. Clin Toxicol 2013; 51: 277. 